\title{
Stepwise endo-/epicardial catheter ablation for atrial fibrillation: The Mediterranea approach
}

\author{
Giuseppe De Martino MD, $\mathrm{PhD}^{1}$ | Paolo Compagnucci MD ${ }^{2}$ (1) | \\ Carmine Mancusi MD ${ }^{1}$ | Enrico Vassallo MD $^{1}$ | Claudia Calvanese MD $^{1}$ | \\ Giuseppe Della Ratta MD ${ }^{1}$ | Mariateresa Librera MD ${ }^{3}$ | Marco Franciulli MD \\ Luigi Marino $\mathrm{MD}^{4}$ | Antonio Dello Russo MD, $\mathrm{PhD}^{2}$ | Michela Casella MD, $\mathrm{PhD}^{5}$ (
}

${ }^{1}$ Arrhythmology and Heart Failure Unit, Mediterranea Hospital, Naples, Italy

${ }^{2}$ Department of Biomedical Sciences and Public Health, Cardiology and Arrhythmology Clinic, University Hospital "Ospedali Riuniti", Marche Polytechnic University, Ancona, Italy

${ }^{3}$ Echocardiography Laboratory, Mediterranea Hospital, Naples, Italy

${ }^{4}$ Cardiac Surgery Unit, Mediterranea Hospital, Naples, Italy

${ }^{5}$ Department of Clinical, Special, and Dental Sciences, Cardiology and Arrhythmology Clinic, University Hospital "Ospedali Riuniti", Marche Polytechnic University, Ancona, Italy

\section{Correspondence}

Paolo Compagnucci, MD, Cardiology and Arrhythmology Clinic, Department of Biomedical Sciences and Public Health, University Hospital "Ospedali Riuniti" and Marche Polytechnic University, Via Tronto 10/a, 60126, Ancona, Italy.

Email: paolocompagnucci1@gmail.com

Disclosures: None.

\begin{abstract}
Background: Outcomes of catheter ablation (CA) among patients with nonparoxysmal atrial fibrillation (AF) are largely disappointing.

Objective: We sought to evaluate the feasibility, effectiveness, and safety of a single-stage stepwise endo-/epicardial approach in patients with persistent/ longstanding-persistent AF.

Methods: We enrolled 25 consecutive patients with symptomatic persistent $(n=4)$ or longstanding-persistent $(n=21) \mathrm{AF}$ and at least one prior endocardial procedure, who underwent CA using an endo-/epicardial approach. Our anatomical stepwise protocol included multiple endocardial as well as epicardial (Bachmann's bundle [BB] and ligament of Marshall ablations) components, and entailed ablation of atrial tachycardias emerging during the procedure. The primary outcome was freedom from any AF/atrial tachycardia episode after a 3-month blanking period. The secondary outcome was patients' symptom status during follow-up.

Results: The stepwise endo-/epicardial approach allowed sinus rhythm restoration in $72 \%$ of patients, either directly $(n=6,24 \%)$ or after AF organization into atrial tachycardia $(n=12,48 \%)$. BB's ablation was commonly implicated in arrhythmia termination. After a median follow-up of 266 days (interquartile range, 96 days), survival free from AF/atrial tachycardia was $88 \%$. Antiarrhythmic drugs could be discontinued in 22 patients (88\%). As compared to baseline, more patients were asymptomatic at 9 -month follow-up ( $0 \%$ vs. $56 \%, p=.02$ ). Five patients (20\%) developed mild medical complications, whereas one subject (4\%) had severe kidney injury requiring dialysis. Conclusion: A single-stage endo-/epicardial CA resulted in favorable rhythm and symptom outcomes in a cohort of patients with symptomatic persistent/
\end{abstract}

Abbreviations: AF, atrial fibrillation; BB, Bachmann's bundle; CA, catheter ablation; EHRA, European Heart Rhythm Association; IQR, interquartile range; LA, left atrium; LOM, ligament of Marshall; PVI, pulmonary vein isolation; RA, right atrium.

Drs. De Martino and Compagnucci shares the first authorship.

This is an open access article under the terms of the Creative Commons Attribution-NonCommercial-NoDerivs License, which permits use and distribution in any medium, provided the original work is properly cited, the use is non-commercial and no modifications or adaptations are made.

(c) 2021 The Authors. Journal of Cardiovascular Electrophysiology published by Wiley Periodicals LLC 
longstanding-persistent $\mathrm{AF}$ and one or more prior endocardial procedures. Epicardial ablation of BB was commonly implicated in procedural success.

\section{KEYWORDS}

atrial fibrillation, Bachmann's bundle, catheter ablation, endocardial ablation, epicardial ablation

\section{1 | INTRODUCTION}

Catheter ablation (CA) is a pivotal component of the rhythm control strategy in patients with symptomatic atrial fibrillation (AF). ${ }^{1,2}$ Pulmonary vein isolation (PVI) is well established as the cornerstone procedural target in case of a first AF ablation, due to the prominent role played by pulmonary veins in arrhythmia initiation in paroxysmal AF. ${ }^{1,2}$

However, procedural outcomes are still suboptimal in patients with persistent $A F$, and in these subjects additional anatomical structures and/or electrophysiological triggers are commonly ablated on top of PVI, in an attempt to improve ablation effectiveness. ${ }^{2-5}$

In recent years, we have witnessed a renewed interest in atrial anatomy and in the role of epicardial structures, such as Bachmann's bundle (BB) and the ligament of Marshall (LOM), as critical determinants of $A F{ }^{6,7}$ At the same time, percutaneous epicardial ablation has gained popularity as an important component of the therapeutic armamentarium for ventricular tachycardia. ${ }^{8}$

Driven by the desire to address arrhythmogenic epicardial structures while avoiding complications related to a thoracotomic approach, minimally invasive epicardial ablation for AF has now entered the clinical arena, and some preliminary experiences supported its feasibility and allowed a greater understanding of the transmurality of the AF substrate, with encouraging clinical results. ${ }^{9-11}$

Based on these preliminary observations, we developed a combined endo-/epicardial stepwise protocol for AF CA in patients with persistent or longstanding persistent $\mathrm{AF}$ and one or more prior endocardial procedures, the Mediterranea approach. In the present study, we describe our protocol in detail, focusing on technical aspects of the epicardial approach to relevant atrial structures, providing insights into the sites of $A F$ termination, and reporting clinical outcomes.

\section{2 | METHODS}

\subsection{Design and inclusion/exclusion criteria}

We conducted a single-center, prospective, observational study at the Arrhythmology Unit of the Mediterranea Center, Naples, Italy, enrolling consecutive patients with symptomatic persistent or longstanding persistent $\mathrm{AF}$, who were referred for endo-/epicardial CA after at least one prior endocardial procedure. Exclusion criteria included prior cardiac surgery or other pericardial diseases predisposing to pericardial adhesions.

The study was carried out according to institutional standards, national legal requirements, and the Declaration of Helsinki. Informed consent was obtained from all patients. Being an observational study, approval of the ethics committee was waived.

\subsection{Endpoints and follow-up}

The primary endpoint of our study was freedom from any AF/ atrial tachycardia episode lasting more than $30 \mathrm{~s}$ after the endo-/ epicardial CA. AF/atrial tachycardia occurring within the first 3 months after the procedure (also known as "blanking period") were not counted in the primary outcome analysis, in accordance with existing guidelines. ${ }^{2}$ The secondary endpoint was the change in the European Heart Rhythm Association (EHRA) score over follow-up. Patients were followed by symptoms, electrocardiography, and 24-h Holter monitor recordings 1, 3, 6, 9, and 12 months after CA; furthermore, arrhythmia recurrences were assessed by implantable loop recorders or pacemakers/defibrillators with atrial leads, when available.

\section{3 | Procedural approach}

Endo-/epicardial CA was performed in the electrophysiology laboratory, under general anesthesia. Patients were studied during AF; in case of sinus rhythm at the beginning of the procedure, AF/atrial tachycardia was induced using burst stimulation.

We performed an extensive ablation procedure in each patient, starting from the LA endocardium, which was accessed with a single transseptal puncture. Unfractionated heparin was administered to maintain activated clotting time values greater than $300 \mathrm{~s}$. Procedures were guided by electroanatomical mapping system (CARTO; Biosense Webster)-derived threedimensional reconstructions of the atria. Biatrial endocardial maps were obtained with a circular mapping catheter (Lasso; Biosense Webster), whereas biatrial epicardial replica were obtained using the ablation catheter (Thermocool Smarttouch; Biosense Webster). CA was guided by ablation index, targeting values higher than 550 in every site, apart from the posterior wall of the left atrium (LA) and the epicardium, where values higher than 500 were obtained. ${ }^{12}$ 
FIGURE 1 Procedural workflow. The stepwise endo-/epicardial atrial fibrillation ablation approach. Endocardial steps are represented in yellow boxes, whereas epicardial steps are represented in orange boxes. LA, left atrium; PVI, pulmonary vein isolation

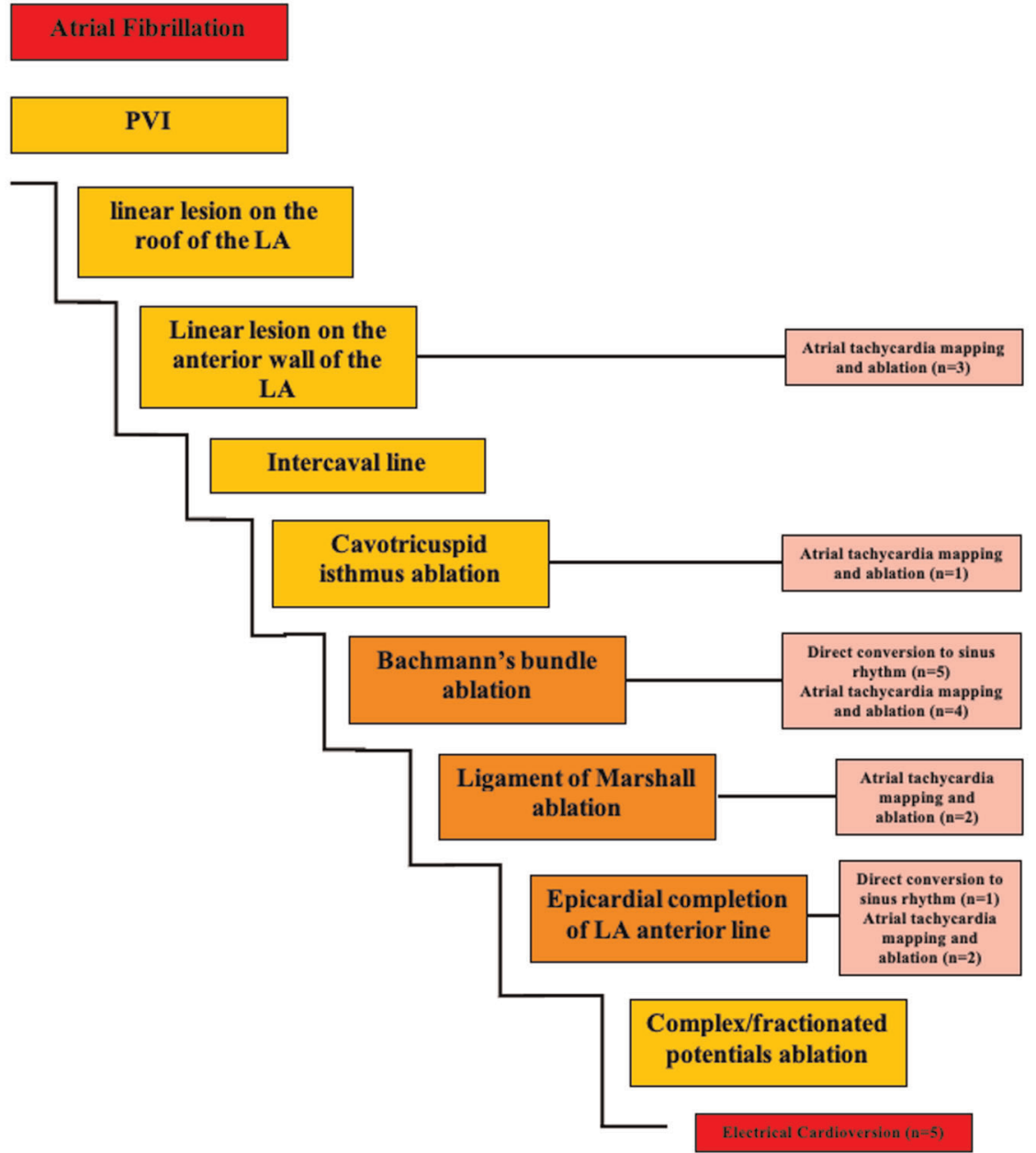

Sinus Rhythm
The procedural approach is represented in Figure 1, and the final lesion set is displayed in Figure 2. The endocardial phase of the procedure aimed at insulating pulmonary veins and closing LA and right atrial anatomical isthmuses. Endocardial steps included antral $\mathrm{PVI}$, followed by linear lesions on the roof of the LA connecting right and left superior pulmonary veins, on the anterior wall of the LA connecting the roof line with mitral valve annulus, in the septal aspect of the posterior wall of the right atrium (RA) connecting superior and inferior venae cavae, and cavotricuspid isthmus ablation.

After completing this lesion set, access was gained to the epicardium, by creating a small $(2-3 \mathrm{~cm})$ pericardial window, through which a steerable introducer (Agilis EPI; Abbott) was placed into the pericardial space. The pericardial window was performed in collaboration with a cardiac surgeon. We preferred such an approach to the classic pericardial puncture described by Sosaosa et al., ${ }^{13}$ because of the more favorable catheter manipulability with the epicardial window.

In the epicardium, BB and the LOM were ablated first according to their anatomic location (in the floor of the transverse sinus close to the interatrial septum, and between the LA appendage and pulmonary veins, respectively), followed by the eventual completion of roof and anterior linear lesions in the proximity of the mitral valve in case of absence of lesion transmurality. In case of ongoing AF, further ablation lesions were delivered in the atria from the endocardium, targeting complex/ fractionated atrial electrograms in both left and right atria. In case patients developed atrial tachycardia at any step during the procedure, the arrhythmia was mapped and ablated, using a highdensity catheter (PentaRay; Biosense Webster). If sinus rhythm was not restored at this point, patients were electrically cardioverted. Bidirectional conduction block across all linear lesions and PV circumferential entrance and exit blocks were verified in sinus rhythm by pacing techniques, as described elsewhere. ${ }^{14} \mathrm{At}$ the end, burst pacing was performed to confirm loss of arrhythmia inducibility from two different sites (coronary sinus and LA appendage), until refractoriness was reached, or an arrhythmia induced. In case of atrial tachycardia induction, the latter was mapped and ablated, aiming to restore sinus rhythm; in case of AF induction, we performed electrical cardioversion. AF termination, lack of arrhythmia inducibility, and validation of conduction block through ablation lines were the acute procedural endpoints. 

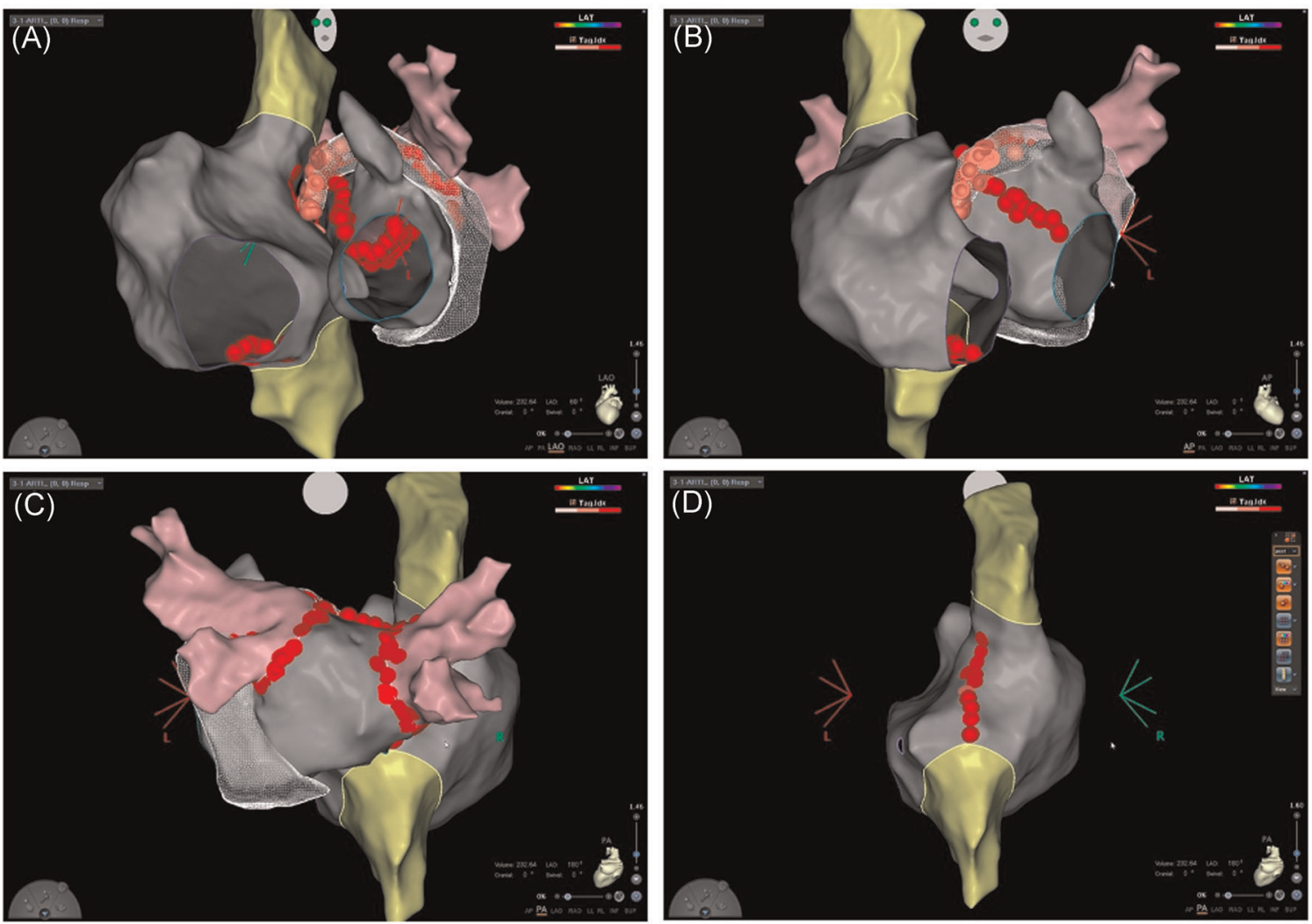

FIGURE 2 Final lesion set. Typical final lesion set of the stepwise endo-/epicardial atrial fibrillation approach; ablation lesions are represented as red tags (endocardial ablations) or rose tags (epicardial ablations). Endocardial biatrial maps are shown in gray (atrial walls), rose (pulmonary veins), or yellow (venae cavae), whereas epicardial map is semi-transparent. (A) Left anterior oblique view; (B) antero-posterior view; (C) biatrial postero-anterior view; (D) right atrial postero-anterior view

To reduce pericardial pain and inflammation, we injected triamcinolone $80 \mathrm{mg}$ directly into the pericardial sac at the end of the procedure; furthermore, we usually administered tramadol $200 \mathrm{mg}$, metoclopramide $20 \mathrm{mg}$, ondansetron $8 \mathrm{mg}$, endovenous dexamethasone $8 \mathrm{mg}$, and a 1-day analgesic infusion pump for the first $24 \mathrm{~h}$ after the procedure. Class III or Ic antiarrhythmic drugs were prescribed for the first three months after CA and suspended thereafter in case of absence of arrhythmia recurrences.

\subsection{Statistical analysis}

Continuous variables were checked for normality using the Shapiro-Wilk test, and are reported as mean and standard deviation if normally distributed, or as median and interquartile range (IQR) if nonnormally distributed. Categorical variables are presented as counts and percentages, and differences between groups were calculated with the two-tailed Fisher's exact test. The primary outcome was assessed in a time-to-event analysis, according to the Kaplan-Meier method. The EHRA scores at baseline and at 9-month follow-up were compared with the Wilcoxon rank-sum test for repeated measures. A twp-sided $p<.05$ defined statistical significance. All statistical analyses were performed with the software RStudio (RStudio Inc).

\section{RESULTS}

We included 25 consecutive patients who underwent endo-/ epicardial AF CA since September 2019 until February 2020.

The characteristics of the patients at baseline are presented in Table 1. The most common indication for CA was longstanding persistent AF $(n=21,84 \%)$. Patients presented with a significant symptom burden (median EHRA class, 3; IQR, 2), after having tried a median of two antiarrhythmic medications (IQR, 1), including amiodarone in 22 patients (88\%). Each subject $(n=25)$ had previously undergone PVI once, 10 patients (40\%) twice, whereas LA roof and mitral isthmus lines had been performed in 5 (20\%) and 2 (8\%) patients, respectively. The mean LA volume was $161 \mathrm{ml}$ (IQR, 25), whereas the median left ventricular ejection fraction measured 54\% (IQR, 10).

\subsection{Primary and secondary endpoints}

After a median follow-up of 266 days (IQR, 96 days), AF/atrial tachycardia-free survival was $88 \%$ (Figure 3). Among the three subjects experiencing recurrences (12\%), one had persistent atrial tachycardia, whereas the remaining two had both persistent $\mathrm{AF}$ and atrial tachycardia over the course of follow-up. 
TABLE 1 Characteristics of the patients at baseline

\begin{tabular}{|c|c|}
\hline & $\begin{array}{l}\text { Overall } \\
(n=25)\end{array}$ \\
\hline Age-years (SD) & $60(9)$ \\
\hline Male sex-no. (\%) & $17(68)$ \\
\hline EHRA score-no. (IQR) & $3(2)$ \\
\hline $\mathrm{CHA}_{2} \mathrm{DS}_{2}$-VASc score-no. (IQR) & $2(2)$ \\
\hline Long-standing persistent atrial fibrillation-no. (\%) & $21(84)$ \\
\hline $\mathrm{BMI}-\mathrm{kg} / \mathrm{m}^{2}(\mathrm{SD})$ & $28(5)$ \\
\hline Hypertension-no. (\%) & $19(76)$ \\
\hline Diabetes-no. (\%) & $4(16)$ \\
\hline Coronary artery disease-no. (\%) & $5(20)$ \\
\hline Heart failure-no. (\%) & $10(40)$ \\
\hline LVEF-\% (IQR) & $54(10)$ \\
\hline LA volume-ml (SD) & $161(25)$ \\
\hline LA diameter-mm (SD) & $49(5)$ \\
\hline Interventricular septum thickness-mm (SD) & $12(3)$ \\
\hline Duration of last AF episode-months (IQR) & $7(4)$ \\
\hline Duration since first AF episode-months (SD) & $49(21)$ \\
\hline Previously failed AADs-no (IQR) & $2(1)$ \\
\hline Prior single PVI-no. (\%) & $15(60)$ \\
\hline Prior double PVI-no. (\%) & $10(40)$ \\
\hline Prior left atrial roof line-no. (\%) & $5(20)$ \\
\hline Prior mitral isthmus line-no. (\%) & $2(8)$ \\
\hline $\begin{array}{l}\text { Pulmonary vein reconnection at the time of } \\
\text { endo-epicardial catheter ablation-no. (\%) }\end{array}$ & $12(48)$ \\
\hline
\end{tabular}

Note: Continuous variables are expressed as mean (standard deviation) when normally distributed, or as median (interquartile range) when nonnormally distributed. Categorical variables are expressed as number (\%).

Abbreviations: $A A D$, antiarrhythmic drug; $A F$, atrial fibrillation; $B M I$, body mass index; EHRA, European Heart Rhythm Association; IQR, interquartile range; LA, left atrial; LVEF, left ventricular ejection fraction; $\mathrm{PVI}$, pulmonary vein isolation.

The primary outcome was assessed by means of loop recorders in seven (28\%) and pacemakers/defibrillators in three patients (12\%), respectively. Antiarrhythmic drugs were discontinued 3 months after CA in 22 subjects (88\%); the three subjects who remained on antiarrhythmic medications were treated with amiodarone. The AF/atrial tachycardia free survival off-antiarrhythmic drugs measured $80 \%$.

The EHRA score significantly improved from baseline to 9month follow-up (median EHRA score at baseline, 3 [IQR, 2]; median EHRA score at 9-month follow-up, 1 [IQR, 1]; $p=.03$; Figure 4), and the proportion of patients with no symptoms (EHRA class I) significantly increased during the same time interval (0/25 at baseline, 14/25 at 9-month follow-up; $p=.02)$.

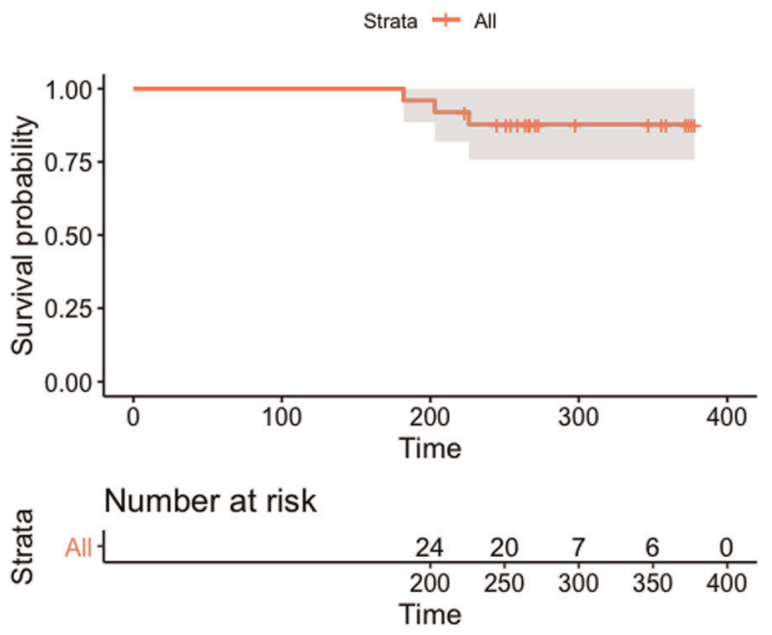

FIGURE 3 Survival free from atrial fibrillation/atrial tachycardia recurrence

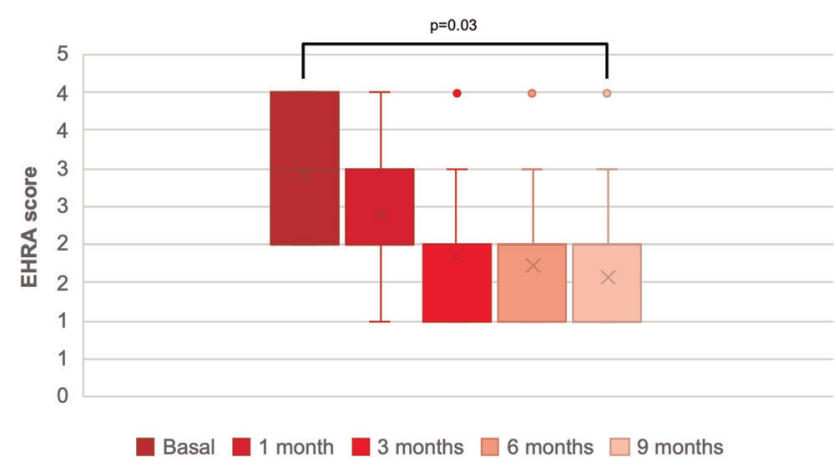

FIG URE 4 European Heart Rhythm Association (EHRA) score during follow-up. EHRA, European Heart Rhythm Association

\section{2 | Procedural characteristics and outcomes}

Patients' baseline rhythm on the day of the procedure was AF in 22 cases (88\%), whereas one patient had atrial tachycardia, and three patients were in sinus rhythm. In two of these latter, AF could be induced with burst stimulation. Of note, 12 patients (48\%) had pulmonary vein reconnection at the time of endo-/epicardial CA.

During the stepwise procedure, sinus rhythm was restored in 18 patients (72\%). We either observed direct conversion of AF to sinus rhythm during ablation in six patients (24\%), or an initial organization into atrial tachycardias ( $n=33$ atrial tachycardias), which were subsequently effectively mapped and ablated in 12 patients (48\%). Six patients (24\%) required electrical cardioversion at the end of the procedure, whereas in the remaining subject (4\%) AF was noninducible at baseline.

Direct conversions to sinus rhythm most commonly occurred during ablation of the BB $(n=5)$, whereas one patient had sinus rhythm directly restored during ablation of complex/fractionated potentials in the RA, from the endocardium. Pulmonary veins were never implicated in arrhythmia termination. 
Bidirectional conduction block across the linear lesion on the anterior wall of the LA was obtained in 16 patients (64\%) after endocardial CA, whereas in the remaining nine patients (36\%) epicardial lesions were needed.

Notably, at the end of the procedure, lack of arrhythmia inducibility was observed in 21 patients (84\%). Two patients (8\%) developed AF, which was electrically cardioverted, whereas five atrial tachycardias were induced, mapped and ablated in two subjects (8\%), restoring sinus rhythm; however, AF inducibility persisted in these two latter patients.

Overall, during the course of the stepwise procedure, AF organized into a median number of one atrial tachycardia per patient (IQR, 3; total count, 38). Of these arrhythmias, nine (24\%) were mapped and successfully ablated in the BB (Figure 5), seven in the proximal portion of the LA appendage (18\%), and five (13\%) in the free wall of the RA; other locations were rarer (Table 2).

\section{3 | Feasibility of the epicardial approach to the Bachman's bundle}

In each patient, we evaluated the technical feasibility of different approaches to the roof of the LA, where the fibers of BB connecting right and left atria in the epicardium can be found and ablated. The roof of the LA represents the floor of the transverse sinus, a tubular structure enveloped by pericardium which forms during organogenesis. ${ }^{15}$

In all but two cases ( $n=23,92 \%)$, the transverse sinus could be reached from the left, advancing the catheter anteriorly to the lateral ridge (Figure 6). A rightward approach to the transverse sinus was feasible in 12 patients $(48 \%)$ by passing close to the septal portion of right atrial appendage (Figure SA), and in 7 patients passing laterally to the right atrial appendage (28\%; Figure SB). The leftward approach proved to be significantly more feasible than other rightward approaches (Fisher's test $p=.019$ ).

\subsection{Procedural duration and complications}

The median length of the procedure was $360 \mathrm{~min}$ (IQR, $60 \mathrm{~min}$ ), while median total, endocardial, and epicardial ablation times measured 56 (IQR, $21 \mathrm{~min}$ ), 49 (IQR, $19 \mathrm{~min}$ ), and $9 \mathrm{~min}$ (IQR, $5 \mathrm{~min}$ ), respectively. We noticed no intraprocedural complications, and the median duration of hospitalization measured 7 days (IQR, 2 days). Following CA, six patients (24\%) developed medical issues, five of which were mild (mild acute kidney injury, $n=3$; cystitis, $n=1$; pneumonia, $n=1$ ), and were managed with conservative measures. One patient (4\%) developed severe acute kidney injury requiring dialysis, followed by partial recovery in renal function (serum creatinine levels at last follow-up, $1.6 \mathrm{mg} / \mathrm{dl}$ ), with no need for permanent renal replacement therapy. Remarkably, there were no stroke/transient ischemic attacks, and no patient was hospitalized or had urgent visits for heart failure during follow-up.

TABLE 2 Atrial tachycardias mapped and ablated during the procedure $(n=38)$

\begin{tabular}{lll}
$\begin{array}{l}\text { Location of earliest activation/ } \\
\text { arrhythmia type }\end{array}$ & AT count & $\begin{array}{l}\text { Mean CL } \\
\text { (SD), ms }\end{array}$ \\
\hline $\begin{array}{l}\text { Bachmann's bundle } \\
\text { Proximal portion of the LA appendage }\end{array}$ & 7 & $352(58)$ \\
\hline Free wall of the RA & 5 & $351(49)$ \\
\hline Ligament of Marshall & 4 & $391(88)$ \\
\hline Posterior wall of the LA & 4 & $275(56)$ \\
\hline Coronary sinus ostium & 3 & $361(41)$ \\
\hline Perimitral flutter & 3 & $435(95)$ \\
\hline Biatrial flutter & 1 & $233(8)$ \\
\hline Posterior wall of the RA & 1 & 375 \\
\hline High septal region of the RA & 1 & 512 \\
\hline
\end{tabular}

Abbreviations: AT, atrial tachycardia; CL, cycle length; LA, left atrium; RA, right atrium; SD, standard deviation.

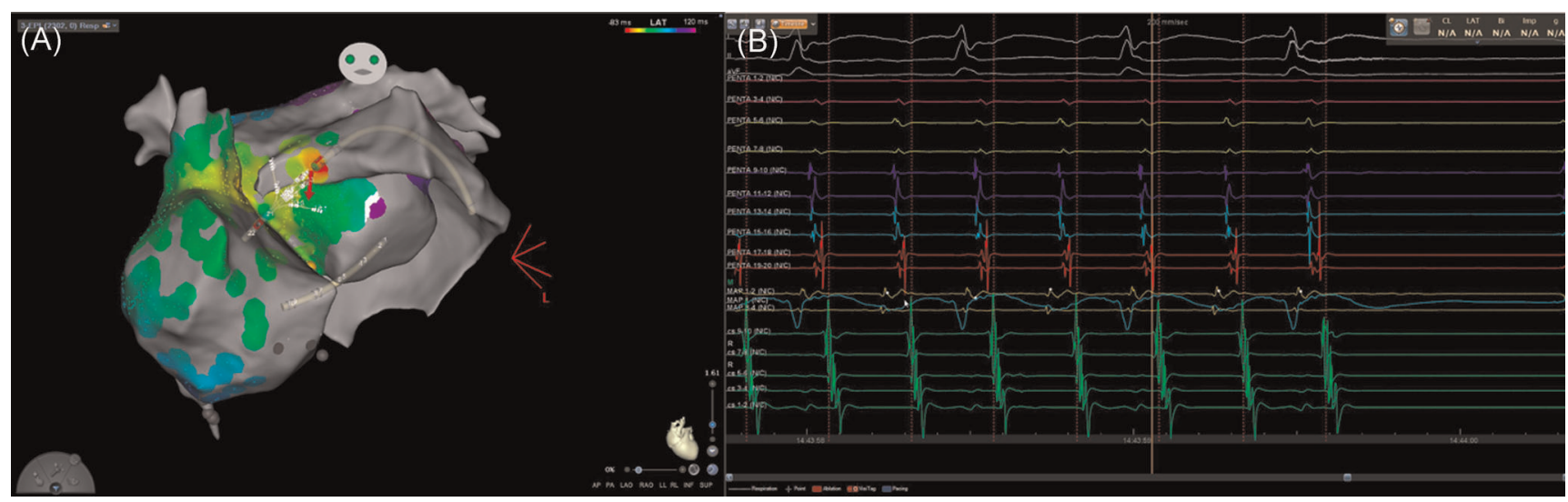

FIGURE 5 Epicardial mapping and ablation of an atrial tachycardia originating from the Bachmann's bundle. Biatrial epicardial activation mapping of an atrial tachycardia appearing during the course of the stepwise endo-/epicardial ablation, antero-posterior view. Note that the site of earliest activation (red spot) is located epicardially, in the anatomical region of the Bachmann's bundle (A); radiofrequency energy delivery in that site resulted in atrial tachycardia termination (B) 

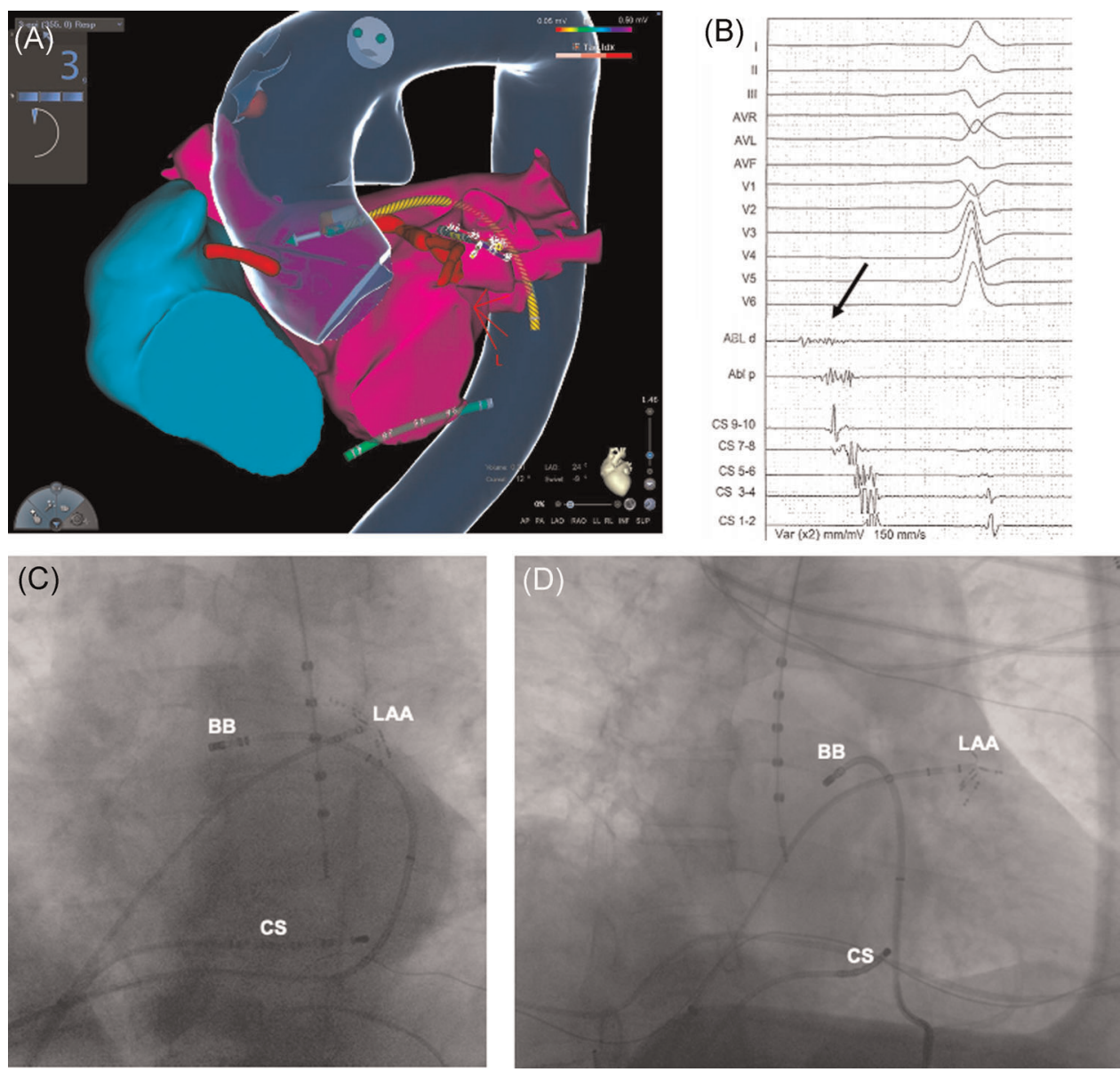

FIGURE 6 Leftward approach to the transverse sinus. (A) Biatrial endocardial electroanatomical reconstruction, left anterior oblique view. Note that the ablation catheter (yellow) is located inside the transverse sinus in the anatomical position of the Bachmann's bundle (BB). The transverse sinus is accessed from the left, passing between the left atrial appendage (LAA) and the pulmonary veins. This access was feasible in 92\% of the study cohort. (B) Low-amplitude fragmented potential (black arrow), typically recorded on the Bachmann's bundle. (C,D) Left anterior oblique (C) and right anterior oblique (D) fluoroscopy projections, showing the ablation catheter inside the transverse sinus from the leftward approach. CS, coronary sinus

\section{4 | DISCUSSION}

In the present study, we described for the first time the feasibility and effectiveness of a novel stepwise endo-/epicardial approach for persistent/longstanding persistent $\mathrm{AF}$ ablation and one or more prior endocardial CA. The key messages are as follows: (1) a stepwise endo-/ epicardial CA approach, by addressing endocardial as well as epicardial atrial arrhythmogenic substrates and ensuring lesion transmurality, could restore sinus rhythm in the vast majority of patients with persistent or longstanding persistent AF; (2) as previously hypothesized, ${ }^{6}$ epicardial ablation of the BB appeared to be a fundamental step of our comprehensive approach, resulting in conversion to sinus rhythm in a high proportion of cases; (3) during follow-up, most of these patients did not experience AF recurrences, allowing the discontinuation of antiarrhythmic drugs; (4) we observed a significant improvement in patients' symptom status during follow-up, and the majority of patients were asymptomatic 9 months after the procedure; (5) our approach resulted in no mechanical complications and in a low risk of medical issues, usually managed with conservative measures; (6) although pericardial reflections and/or sinuses have been classically considered a hindrance to epicardial ablation of arrhythmogenic atrial structures, ${ }^{15}$ catheter manipulation into the transverse sinus was feasible in each patient, and allowed effective $\mathrm{CA}$ of the BB.

The optimal ablation approach for patients with persistent AF has yet to be determined. ${ }^{3-5}$ Recently, endo-epicardial dissociation has been proposed as a key mechanism underlying AF persistence in both animal models and human mapping studies, whereby conduction velocity and direction differ in the various atrial myocardial layers, acknowledging the transmural nature of the fibrillatory process in patients with longstanding AF episodes. ${ }^{16}$

Furthermore, the role of epicardial structures in the pathogenesis of $A F$ is increasingly being recognized. ${ }^{6,7} A$ direct relationship between $\mathrm{BB}$ and $\mathrm{AF}$ is supported by epidemiological, animal, and clinical studies. ${ }^{17}$ Longitudinal dissociation of conduction along BB, by predisposing to re-entrant circuits, is an important mechanistic link between $\mathrm{BB}$ and $\mathrm{AF}{ }^{6}$ Based on these observations, delivering ablation lesions on the BB during AF CA could eliminate a substrate for inhomogeneous conduction and AF perpetuation. ${ }^{18}$ Remarkably, 
given the epicardial location of BB's body, its thickness (a median of $4 \mathrm{~mm}$ ), and the presence of fatty tissue separating it from the limbus of the fossa ovalis, ${ }^{6}$ an endocardial CA approach aiming to eliminate such an arrhythmogenic substrate would only address distal extensions of the interatrial conduction system, leaving the BB's body and its re-entrant circuits substantially unchanged.

Our data support these hypotheses, since epicardial BB's ablation resulted in restoration of sinus rhythm in $20 \%$ of cases, and the BB was also the most common site where atrial tachycardias appearing during the procedure could be mapped. Therefore, epicardial BB's ablation appears to be a fundamental measure to incorporate in a stepwise CA procedure aiming to restore sinus rhythm. Although previous studies raised the concern that BB's ablation could result in impairment of LA mechanical function and increase thromboembolic risk by delaying electrical activation of the LA appendage, ${ }^{6,19,20}$ it is worth reiterating that among patients with persistent/longstanding persistent AF, disrupted interatrial conduction is commonly present at baseline, ${ }^{17}$ confounding the assessment of the impact of CA. In our cohort, in which LA appendage was never electrically isolated, and all patients received long-term anticoagulant treatment as per $\mathrm{CHA}_{2} \mathrm{DS}_{2}$-VASc score, ${ }^{1,2}$ no thromboembolic complication occurred. In addition, as a possible alternative to anticoagulation, LA appendage devices could potentially be used to mitigate stroke risk in these patients. ${ }^{21}$

Closure of a large set of anatomical isthmuses in LA and RA endocardium, as well as BB and LOM ablation in the epicardium resulted in a high rate of $\mathrm{AF}$ organization into atrial tachycardias, whose elimination led to AF termination in a high proportion of our cohort. ${ }^{22}$ This finding suggests that our stepwise approach results in emergence of important drivers sustaining arrhythmia recurrences in the form of atrial tachycardias in the individual patient, allowing a tailored approach aimed at eliminating these drivers. ${ }^{22,23}$ As compared to previous studies, ${ }^{9-11,23}$ we used for the first time an endo-/epicardial strategy to chase each emerging atrial tachycardia. By transmurally mapping and ablating these arrhythmias, we were able to obtain very high procedural and middleterm success. Furthermore, the addition of epicardial ablations allowed us to obtain lesion transmurality and bidirectional conduction block in the roof and anterior LA lines, thus eliminating potential substrates for atrial tachycardias during follow-up., ${ }^{9,23}$

Our stepwise endo-/epicardial approach differs in several ways from previously described hybrid techniques for AF CA. ${ }^{24,25}$ Although we gained access to the pericardial space with a small subxiphoid pericardial window, we did not navigate the pericardium under direct vision, and all ablation lesions were delivered by the electrophysiologist using conventional ablation catheters, therefore limiting the need for assistance by the cardiac surgeon to the initial and terminal moments of the procedure. Furthermore, although every case was performed in co-operation with cardiac surgeons, the pericardial access was successfully performed by the electrophysiologist as first operator in the last 15 patients ablated, suggesting that a mini-invasive pericardial access is not surgically demanding. In addition, use of a percutaneous technique may facilitate the epicardial access further, by eliminating logistical hurdles of co-ordination between electrophysiologists and cardiac surgeons, as shown by other authors. $^{9-11}$ As compared to other hybrid techniques, the ability to deliver all ablation lesions during a single-stage procedure in a transmural fashion is an important advantage of our approach, which appears to be particularly appropriate for symptomatic patients strongly desiring to have their arrhythmia rapidly eliminated.

In patients with persistent $A F$, antiarrhythmic drugs are often prescribed to facilitate rhythm control. ${ }^{1,26}$ However, even amiodarone, the most effective drug, is inferior to CA in maintaining sinus rhythm ${ }^{1,27}$ and is plagued by adverse events, which can sometimes be lifethreatening. ${ }^{1,26}$ Endo-/epicardial CA may represent the ideal therapeutic strategy for younger patients, by allowing discontinuation of antiarrhythmic medications in the vast majority, thus avoiding long-term exposure to amiodarone. ${ }^{26}$

As a final remark, we found that each patient could be discharged in sinus rhythm irrespective of the presence of marked atrial dilation and longstanding AF duration, that most of these patients did not experience recurrences at follow-up, and that only one patient had a serious adverse medical event. These findings might support a more aggressive approach to rhythm control among patients with persistent/longstanding persistent $\mathrm{AF}$ and one or more prior endocardial $\mathrm{CA}$, by incorporating the stepwise endo-/epicardial ablation protocol, which may represent a viable option when adverse atrial remodeling is present.

\section{5 | LIMITATIONS}

We could only enroll 25 patients from a single hospital in our study, with a short median duration of follow-up, and therefore larger numbers of patients enrolled in multiple centers with longer observation times would have been required to derive statistically rigorous results in support of the stepwise endo-/epicardial approach for AF CA. All procedures were performed by expert operators, potentially limiting the generalizability of our findings. The majority of patients did not have cardiac implanted devices, which might have led to an underestimation of subclinical AF recurrences. However, AF/atrial tachycardia recurrences in a cohort of patients with persistent AF and markedly dilated LA could expectably present as non-self-limiting episodes, which would likely be detectable by intermittent rhythm monitoring.

\section{6 | CONCLUSION}

A stepwise percutaneous endo-/epicardial stepwise approach for CA of persistent and longstanding persistent AF resulted in restoration and maintenance of sinus rhythm in the majority of patients at 9-month follow-up. BB ablation appeared to be a pivotal component of such an approach, and was feasible in all patients, with low risk of serious procedural complications. Further studies are highly needed to confirm our promising results, which could represent an important step forward in the management of AF in patients with persistent or longstanding persistent forms of the arrhythmia after a failed endocardial AF CA. 


\section{CONFLICT OF INTERESTS}

The authors declare that there are no conflict of interests.

\section{DATA AVAILABILITY STATEMENT}

Data available on request from the authors.

\section{ORCID}

Paolo Compagnucci (DD http://orcid.org/0000-0003-1924-6548 Michela Casella (D) http://orcid.org/0000-0002-5322-1742

\section{REFERENCES}

1. Hindricks G, Potpara T, Dagres N, et al. ESC guidelines for the diagnosis and management of atrial fibrillation developed in collaboration with the European Association of Cardio-Thoracic Surgery (EACTS). Eur Heart J. 2020;2020:ehaa612.

2. Calkins $\mathrm{H}$, Hindricks $\mathrm{G}$, Cappato R, et al. HRS/EHRA/ECAS/APHRS/ SOLAECE expert consensus statement on catheter and surgical ablation of atrial fibrillation. Heart Rhythm. 2017;201714(10):e275-e444.

3. Verma A, Jiang CY, Betts TR, et al. Approaches to catheter ablation for persistent atrial fibrillation. N Engl J Med. 2015;372(19): 1812-1822.

4. Kirchhof $P$, Calkins $H$. Catheter ablation in patients with persistent atrial fibrillation. Eur Heart J. 2017;38(1):20-26.

5. Gianni C, Mohanty S, Trivedi C, Di Biase L, Natale A, Natale A. Novel concepts and approaches in ablation of atrial fibrillation: the role of non-pulmonary vein triggers. Europace. 2018;20(10):1566-1576.

6. van Campenhout MJ, Yaksh A, Kik C, et al. Bachmann's bundle: a key player in the development of atrial fibrillation? Circ Arrhythm Electrophysiol. 2013;6(5):1041-1046.

7. Rodríguez-Mañero M, Schurmann $P$, Valderrábano M. Ligament and vein of Marshall: a therapeutic opportunity in atrial fibrillation. Heart Rhythm. 2016;13(2):593-601.

8. Della Bella P, Brugada J, Zeppenfeld K, et al. Epicardial ablation for ventricular tachycardia: a European multicenter study. Circ Arrhythm Electrophysiol. 2011;4(5):653-659.

9. Piorkowski C, Kronborg M, Hourdain J, et al. Endo-/epicardial catheter ablation of atrial fibrillation: feasibility, outcome, and insights into arrhythmia mechanisms. Circ Arrhythm Electrophysiol. 2018; 11(2):e005748.

10. Jiang R, Buch E, Gima J, et al. Feasibility of percutaneous epicardial mapping and ablation for refractory atrial fibrillation: insights into substrate and lesion transmurality. Heart Rhythm. 2019;16(8): 1151-1159.

11. Yuu L, Liu Q, Jiang RH, et al. Adjunctive percutaneous ablation targeting epicardial arrhythmogenic structures in patients of atrial fibrillation with recurrence after multiple procedures. J Cardiovasc Electrophysiol. 2020;31(2):401-409.

12. Hussein A, Das M, Riva S, et al. Use of ablation index-guided ablation results in high rates of durable pulmonary vein isolation and freedom from arrhythmia in persistent atrial fibrillation patients: the PRAISE study results. Circ Arrhythm Electrophysiol. 2018;11(9): e006576.

13. Sosa E, Scanavacca M, d'Avila A, Pilleggi F, Pilleggi F. A new technique to perform epicardial mapping in the electrophysiology laboratory. J Cardiovasc Electrophysiol. 1996;7(6):531-536.

14. Shah D, Haïssaguerre $M$, Takahashi A, et al. Differential pacing for distinguishing block from persistent conduction through an ablation line. Circulation. 2000;102(13):1517-1522.

15. Lachman N, Syed FF, Habib A, et al. Correlative anatomy for the electrophysiologist, Part I: the pericardial space, oblique sinus, transverse sinus. J Cardiovasc Electrophysiol. 2010;21(12):1421-1426.
16. de Groot N, van der Does L, Yaksh A, et al. Direct proof of endoepicardial asynchrony of the atrial wall during atrial fibrillation in humans. Circ Arrhythm Electrophysiol. 2016;9(5):e003648.

17. Bayés de Luna A, Cladellas M, Oter R, et al. Interatrial conduction block and retrograde activation of the left atrium and paroxysmal supraventricular tachyarrhythmia. Eur Heart J. 1988;9(10): 1112-1118.

18. Moseroser F, Rieger A, Pönisch C, Kottkamp H, Kottkamp H. A novel ablation approach in atrial fibrillation patients undergoing fibrotic-based substrate modification: targeting the Bachmann's bundle? J Cardiovasc Electrophysiol. 2018;29(6):844-853.

19. Zhang J, Tang C, Zhang Y, Su XI, Su XI. Electrophysiologic and clinical consequences of left atrial anterior wall linear ablation in patients with persistent atrial fibrillation. J Cardiovasc Electrophysiol. 2015;26(9):934-943.

20. Rillig A, Tilz RR, Lin T, et al. Unexpectedly high incidence of stroke and left atrial appendage thrombus formation after electrical isolation of the left atrial appendage for the treatment of atrial tachyarrhythmias. Circ Arrhythm Electrophysiol. 2016;9(5):e003461.

21. Glikson M, Wolff R, Hindricks $G$, et al. EHRA/EAPCl expert consensus statement on catheter-based left atrial appendage occlusion-an update [published online ahead of print August 31, 2019]. Europace. https://doi.org/10.1093/europace/euz258

22. Limim HS, Derval N, Komatsu Y, et al. Is ablation to termination the best strategy for ablation of persistent atrial fibrillation? Persistent atrial fibrillation is best ablated by a strategy that terminates the arrhythmia: procedural termination is associated with improved long-term outcomes. Circ Arrhythm Electrophysiol. 2015;8(4): 963-971.

23. Scherr D, Khairy P, Miyazaki S, et al. Five-year outcome of catheter ablation of persistent atrial fibrillation using termination of atrial fibrillation as a procedural endpoint. Circ Arrhythm Electrophysiol. 2015; 8(1):18-24.

24. Khoynezhad A, Ellenbogen KA, Al-Atassi T, et al. Hybrid atrial fibrillation ablation: current status and a look ahead. Circ Arrhythm Electrophysiol. 2017;10(10):e005263.

25. Delurgioe DB, Crossen KJ, Gill J, et al. Hybrid convergent procedure for the treatment of persistent and long standing persistent atrial fibrillation: results of CONVERGE clinical trial. Circ Arrhythm Electrophysiol. 2020;13:009288. https://doi.org/10.1161/CIRCEP. 120.009288

26. Zimetbaum P. Antiarrhythmic drug therapy for atrial fibrillation. Circulation. 2012;125(2):381-389.

27. Di Biase L, Mohanty P, Mohanty S, et al. Ablation versus amiodarone for treatment of persistent atrial fibrillation in patients with congestive heart failure and an implanted device: results from the AATAC multicenter randomized trial. Circulation. 2016;133(17):1637-1644.

\section{SUPPORTING INFORMATION}

Additional Supporting Information may be found online in the supporting information tab for this article.

How to cite this article: De Martino G, Compagnucci P, Mancusi C, et al. Stepwise endo-/epicardial catheter ablation for atrial fibrillation: The Mediterranea approach. J Cardiovasc Electrophysiol. 2021;32:2107-2115. https://doi.org/10.1111/jce.15151 